\title{
Comportamiento de una mezcla densa en CALIENTE ELABORADA CON ASFALTOS MODIFICADOS CON ASFALTITA
}

\author{
Hugo Alexander Rondón Quintana ${ }^{1}$ \\ Fredy Alberto Reyes ${ }^{2}$
}

\section{Resumen}

El presente trabajo evaluó en laboratorio el cambio en las propiedades mecánicas que experimenta una mezcla asfáltica densa en caliente (tipo MDC-2 acorde con INVIAS, 2007) cuando se adiciona por vía húmeda al cemento asfáltico una asfaltita proveniente de la Mina San Alberto (Santander, Colombia). Las propiedades evaluadas fueron la resistencia mecánica bajo carga monotónica, módulo resiliente y resistencia a la deformación permanente. Dos tipos de cemento asfáltico (CA) fueron modificados: CA 80-100 proveniente de la refinería de Barrancabermeja (Colombia) y CA 60-70 proveniente de Apiay (Colombia). De los resultados obtenidos se concluye que la resistencia mecánica bajo carga monotónica, el módulo resiliente y la resistencia a la deformación permanente de mezclas asfálticas tipo MDC-2 modificadas con asfaltita es mayor en comparación con las convencionales (mezclas que emplean asfaltos sin ningún aditivo).

Adicionalmente, se realizaron ensayos de penetración a diferentes temperaturas y punto de ablandamiento sobre los cementos asfálticos con y sin aditivo. Agregando asfaltita al cemento asfáltico se obtiene un material con mayor resistencia a la penetración y menor susceptibilidad térmica a fluir.

1 Ingeniero Civil, Magíster en Ingeniería Civil y Doctor en Ingeniería. Vicedecano de Investigaciones, Facultad de Ingeniería, Universidad Católica de Colombia. e-mail: harondon@ucatolica.edu.co,

2 Ingeniero Civil, Magíster en Ingeniería Civil y Doctor en Ingeniería. Director Maestría en Ingeniería civil, Facultad de Ingeniería, Pontificia Universidad Javeriana. e-mail: fredy.reyes@javeriana.edu.co

Fecha de recepción: 3 de abril de 2009

Fecha de aceptación: 11 de mayo de 2009 


\section{Palabras clave}

Asfalto modificado, mezcla densa en caliente, asfaltita.

\section{Abstract}

Laboratory tests were used to evaluate the effect on the mechanical properties of a hot asphalt mix (MDC-2 as per INVIAS, 2007 specifications) due to the addition by wet way of a natural asphaltite from the San Alberto Mine (Santander, Colombia). The strength under monotonic load, resilient modulus and rutting were evaluated. Two asphalt cements (CA) were used, CA 80-100 from the Barrancabermeja refinery (Colombia) and CA 60-70 from Apiay (Colombia). The results show that the mechanical properties evaluated were higher for the MDC2 mixes modified with asphaltite compared with mixtures with asphalts without additives. Additionally penetration tests at different temperatures and softening points were conducted on asphalt cements with and without additive. The asphaltite produces higher penetration resistance and lower thermal flow susceptibility.

\section{Key words}

Modified Asphalt, hot dense mix, asphaltite. 


\section{INTRODUCCIÓN}

En Colombia, la tendencia del parque automotor en los últimos treinta años ha sido incrementar el número y magnitud de cargas (MINTRANSPORTE - Ministerio de Transporte, 2000, 2004). Lo anterior genera en las capas asfálticas mayores niveles de esfuerzo y deformación. Estos mayores niveles de carga deben ser contrarrestados con materiales asfálticos que presenten mejores comportamientos que los tradicionales. Con el fin de mejorar las propiedades de los materiales asfálticos y las mezclas asfálticas, se han adelantado varias investigaciones en Colombia y el mundo (estados del conocimiento sobre el tema pueden ser consultados en Papagiannakis \& Lougheed, 1995; Copeland, 2007, y para el caso colombiano en Rondón et al, 2008).

El objetivo principal de estas investigaciones ha sido mejorar las propiedades mecánicas, químicas y reológicas de los asfaltos y las mezclas asfálticas convencionales con el fin de elevar la rigidez, la resistencia a carga monotónica, ahuellamiento, fatiga y envejecimiento, y disminuir la susceptibilidad térmica.

$\mathrm{El}$ artículo presenta los resultados experimentales de ensayar una mezcla asfáltica densa en caliente (tipo MDC-2 de acuerdo a INVIAS, 2007) modificada con una asfaltita proveniente de Santander (Colombia). Para la elaboración de las mezclas y la evaluación de las mismas, fueron modificados los dos tipos de cementos asfálticos (CA) fabricados en Colombia: CA 80-100 proveniente de la refinería de Barrancabermeja y CA 60-70 proveniente del municipio de Apiay. La asfaltita se adicionó al CA por vía húmeda a una temperatura adecuada y controlando el tiempo de mezcla para garantizar su homogeneidad. Para la evaluación de la resistencia mecánica bajo carga monotónica de las mezclas asfálticas convencionales y modificadas se empleó el ensayo Marshall y para el CA con y sin aditivo se realizaron ensayos de penetración a diferentes temperaturas y punto de ablandamiento. Con el fin de evaluar el comportamiento de las mezclas bajo carga cíclica se realizaron ensayos de módulo resiliente y deformación permanente medida en la dirección vertical. 


\section{Metodología}

El agregado pétreo empleado para la elaboración de las mezclas asfálticas convencionales y modificadas con asfaltita (llamadas briquetas) es procedente de la cantera "Subachoque" Cundinamarca, Colombia. A estos materiales se le realizaron los siguientes ensayos, siguiendo las especificaciones del Instituto Nacional de Vías (INVIAS, 2007a):

- Análisis granulométrico de agregados gruesos y finos (INV. E-213)

- Peso específico y absorción de agregados finos (INV. E-222), peso específico y absorción de agregados gruesos (INV. E-223)

- Resistencia al desgaste de los agregados (tamaños menores de 3/4") por medio de la máquina de Los Ángeles (INV. E-218)

- Desgaste Micro-Deval (INV. E-238)

- Pérdida en ensayo de solidez (INV. E-220)

- Partículas fracturadas (INV. E-227)

- Ensayo para medir el equivalente de arena (INV. E-133)

- Índice de alargamiento aplanamiento (INV. E-230)

Los CA empleados en el proyecto provienen de las refinerías de la Empresa Colombiana de Petróleos (ECOPETROL) situada en la ciudad de Barrancabermeja y el municipio de Apiay, en Colombia. Las características de los CA se presentan en las Tablas 1 y 2 .

Luego de realizar los ensayos al agregado pétreo se fabricaron cinco briquetas (compactadas a 75 golpes por cara) para cada porcentaje de asfalto entre $4.5 \%$ y $6.5 \%$, con el fin de realizar el diseño Marshall para determinar el contenido óptimo de asfalto de las mezclas convencionales (el diseño se realizó basado en los criterios presentados en la Tabla 3 para el caso de MDC-2). Una vez se obtuvo el porcentaje óptimo de CA, se fabricaron nuevas briquetas agregando por vía húmeda la asfaltita en porcentajes de $0.5,1.5,2.5$ y $3.5 \%$ (con respecto al peso total de la briqueta de 1200 g), manteniendo el porcentaje de CA. Por cada porcentaje de 
aditivo se fabricaron cinco briquetas para ensayarlas en el aparato Marshall. Además se realizó el mismo estudio aumentando y rebajando el porcentaje óptimo de CA en $0.3 \%$.

Tabla 1. Características generales del cemento asfáltico CA 80-100 PROVENIENTE DE BARRANCABERMEJA.

\begin{tabular}{|c|c|c|c|c|}
\hline Ensayo & Método & Unidad & CA 80-100 & Resultado \\
\hline \multicolumn{5}{|c|}{ Ensayos sobre el asfalto original } \\
\hline $\begin{array}{l}\text { Penetración }\left(25^{\circ} \mathrm{C},\right. \\
100 \mathrm{~g}, 5 \mathrm{~s})\end{array}$ & ASTM D-5 & $0.1 \mathrm{~mm}$ & $80-100$ & 85 \\
\hline Índice de penetración & INV. E-724 & - & $-1 /+1$ & -0.5 \\
\hline $\begin{array}{l}\text { Viscosidad absoluta } \\
\left(60^{\circ} \mathrm{C}\right)\end{array}$ & ASTM D-4402 & Poises & 1000 mín. & 1400 \\
\hline $\begin{array}{l}\text { Ductilidad }\left(25^{\circ} \mathrm{C},\right. \\
5 \mathrm{~cm} / \mathrm{min})\end{array}$ & ASTM D-113 & $\mathrm{cm}$ & 100 mín. & $>105$ \\
\hline $\begin{array}{l}\text { Solubilidad en } \\
\text { Tricloroetileno }\end{array}$ & ASTM D-2042 & $\%$ & 99 mín. & $>99$ \\
\hline Contenido de agua & ASTM D-95 & $\%$ & 0.2 máx. & $<0.2$ \\
\hline $\begin{array}{l}\text { Punto de inflamación } \\
\text { COC }\end{array}$ & ASTM D-92 & ${ }^{\circ} \mathrm{C}$ & 232 mín. & 295 \\
\hline \multicolumn{5}{|c|}{ Ensayos sobre el residuo luego del RTFOT } \\
\hline Pérdida de masa & ASTM D-2872 & $\%$ & 1.0 máx. & 0.2 \\
\hline $\begin{array}{l}\text { Penetración del } \\
\text { residuo }\left(25^{\circ} \mathrm{C}, 100 \mathrm{~g} \text {, }\right. \\
5 \mathrm{~s}) \text { con respecto a la } \\
\text { original }\end{array}$ & ASTM D-5 & $\%$ & 48 mín. & 65 \\
\hline
\end{tabular}


Tabla 2. Características generales del cemento asfáltico CA 60-70 PROVENIENTE DE APIAY.

\begin{tabular}{|c|c|c|c|c|}
\hline Ensayo & Método & Unidad & CA $60-70$ & Resultado \\
\hline \multicolumn{5}{|c|}{ Ensayos sobre el asfalto original } \\
\hline $\begin{array}{l}\text { Penetración } \\
\left(25^{\circ} \mathrm{C}, 100 \mathrm{~g}, 5 \mathrm{~s}\right)\end{array}$ & ASTM D-5 & $0.1 \mathrm{~mm}$ & $60-70$ & 67 \\
\hline Índice de penetración & INV. E-724 & - & $-1 /+1$ & -0.7 \\
\hline Viscosidad absoluta $\left(60^{\circ} \mathrm{C}\right)$ & ASTM D-4402 & Poises & 1500 mín. & 1750 \\
\hline Ductilidad $\left(25^{\circ} \mathrm{C}, 5 \mathrm{~cm} / \mathrm{min}\right)$ & ASTM D-113 & $\mathrm{cm}$ & 100 mín. & $>105$ \\
\hline $\begin{array}{l}\text { Solubilidad en } \\
\text { Tricloroetileno }\end{array}$ & ASTM D-2042 & $\%$ & 99 mín. & $>99$ \\
\hline Contenido de agua & ASTM D-95 & $\%$ & 0.2 máx. & $<0.2$ \\
\hline Punto de inflamación COC & ASTM D-92 & ${ }^{\circ} \mathrm{C}$ & 232 mín. & 275 \\
\hline \multicolumn{5}{|c|}{ Ensayos sobre el residuo luego del RTFOT } \\
\hline Pérdida de masa & ASTM D-2872 & $\%$ & 1.0 máx. & 0.4 \\
\hline Viscosidad absoluta $\left(60^{\circ} \mathrm{C}\right)$ & ASTM D-4402 & Poises & $\begin{array}{l}10000 \\
\text { máx. }\end{array}$ & 5200 \\
\hline Índice de durabilidad & - & - & 3 máx. & $<3$ \\
\hline $\begin{array}{l}\text { Penetración del residuo } \\
\left(25^{\circ} \mathrm{C}, 100 \mathrm{~g}, 5 \mathrm{~s}\right) \text { con } \\
\text { respecto a la original }\end{array}$ & ASTM D-5 & $\%$ & 52 mín. & 70 \\
\hline
\end{tabular}


Tabla 3. Criterios de diseño Marshall

PARA MEZCLAS ASFÁLTICAS DENSAS EN CALIENTE.

\begin{tabular}{|c|c|c|c|}
\hline CARACTERÍSTICAS & \multicolumn{3}{|c|}{$\begin{array}{l}\text { TRÁNSITO DE DISEÑO (N) } \\
\text { Ejes equivalentes de } 80 \mathrm{kN}\end{array}$} \\
\hline TRÁNSITO & $\begin{array}{c}>5 \times 10^{6} \\
\quad(\mathrm{NT} 3)\end{array}$ & $\begin{array}{l}5 \times 10^{5}- \\
5 \times 10^{6} \\
(\mathrm{NT} 2)\end{array}$ & $\begin{array}{c}<5 \times 10^{5} \\
(\mathrm{NT} 1)\end{array}$ \\
\hline Compactación, golpes/cara & 75 & 75 & 50 \\
\hline Estabilidad mínima kg & 900 & 750 & 500 \\
\hline Flujo mm & $2-3.5$ & $2-4$ & $2-4$ \\
\hline Vacíos con aire: & & & \\
\hline Capa de rodadura \% & $4-6$ & $3-5$ & $3-5$ \\
\hline Intermedia \% & $4-7$ & $4-8$ & $4-8$ \\
\hline Base asfáltica \% & $5-8$ & $5-9$ & - \\
\hline $\begin{array}{l}\text { Vacíos mínimos en agregados } \\
\text { minerales: }\end{array}$ & & & \\
\hline Mezclas $0 \%$ & $\geq 13$ & $\geq 13$ & $\geq 13$ \\
\hline Mezclas $1 \%$ & $\geq 14$ & $\geq 14$ & $\geq 14$ \\
\hline Mezclas $2 \%$ & $\geq 15$ & $\geq 15$ & $\geq 15$ \\
\hline Mezclas $3 \%$ & $\geq 16$ & $\geq 16$ & $\geq 16$ \\
\hline Vacíos llenos de asfalto \% & $65-75$ & $65-78$ & $65-80$ \\
\hline Relación Estabilidad/Flujo kg/mm & $300-600$ & $300-500$ & $200-400$ \\
\hline
\end{tabular}

Fuente: INVIAS (2007). Especificaciones generales de construcción de carreteras. Artículo 450.4.2.2.

El ensayo de módulo resiliente (INV. E-749, INVIAS, 2007) se realizó empleando tres temperaturas $\left(10,20\right.$ y $\left.30^{\circ} \mathrm{C}\right)$ y frecuencias de carga diferentes $(2.5,5.0$ y $10.0 \mathrm{~Hz})$. El ensayo de resistencia a la deformación permanente bajo carga repetida fue realizado bajo un esfuerzo de $100 \mathrm{kPa}$ y a 3600 ciclos de carga siguiendo el procedimiento normalizado por el prEN 12697-00. Estos ensayos fueron medidos sobre muestras fabricadas con CA 80-100 utilizando el porcentaje óptimo de CA obtenido del ensayo Marshall. Por último se realizaron ensayos de penetración (INV. E - 706) al CA convencional y al modificado siguiendo los procedimientos recomendados por INVIAS (2007a). 
Una descripción detallada de la asfaltita utilizada puede ser consultada en INVIAS (1997). La asfaltita presenta un peso específico de $1.10 \mathrm{~g} / \mathrm{cm}^{3}$ y partículas de coloración negra brillante que pasan el tamiz No. 40 en un ensayo de granulometría por tamizado.

Las temperaturas de mezclado del CA con la asfaltita, y de compactación de las briquetas fueron de $145^{\circ} \mathrm{C}$ y $135^{\circ} \mathrm{C}$ respectivamente (basados en los resultados de los ensayos de viscosidad). El tiempo de mezclado del CA con la asfaltita fue 45 minutos para porcentajes de adición de asfaltita de $0.5 \%$ y $1.5 \%$ y de una hora para porcentajes de $2.5 \%$ y $3.5 \%$.

\section{Resultados y análisis}

Caracterización de los agregados pétreos. Para cumplir con las especificaciones del INVIAS (2007), se modificó la granulometría original de los agregados, tomando como referencia los valores promedios en porcentajes de la franja granulométrica que exige la especificación para la elaboración de las briquetas del ensayo Marshall (Tabla 4). En la Tabla 5 se presentan los valores obtenidos de los ensayos de caracterización al agregado pétreo. Se observa en esta Tabla que los valores de cada uno de los ensayos cumplen con el requisito mínimo de calidad exigido por las especificaciones INVIAS (2007) para fabricar mezclas tipo MDC-2 y,por lo tanto, el material puede ser empleado para la elaboración de las briquetas del presente estudio. 
Tabla 4. Gradación de mezclas densas en Caliente tipo 2 (MDC-2).

\begin{tabular}{ccc}
\hline \multicolumn{2}{c}{ TAMIZ } & PORCENTAJE QUE PASA \\
\hline Normal & Alterno & MDC-2 \\
\hline $25.0 \mathrm{~mm}$ & $1 ”$ & - \\
$19.0 \mathrm{~mm}$ & $3 / 4 ”$ & 100 \\
$12.5 \mathrm{~mm}$ & $1 / 2 "$ & $80-95$ \\
$9.5 \mathrm{~mm}$ & $3 / 8 ”$ & $70-88$ \\
$4.75 \mathrm{~mm}$ & No.4 & $49-65$ \\
$2.00 \mathrm{~mm}$ & No.10 & $29-45$ \\
$425 \mu \mathrm{m}$ & No.40 & $14-25$ \\
$180 \mu \mathrm{m}$ & No.80 & $8-17$ \\
$75 \mu \mathrm{m}$ & No.200 & $4-8$ \\
\hline
\end{tabular}

Fuente: Instituto Nacional de Vías - INVIAS (2007). Especificaciones generales de construcción de carreteras. Artículo 450.2.1.

Tabla 5. Caracterización del agregado pétreo.

\begin{tabular}{lc}
\hline \multicolumn{1}{c}{ ENSAYO } & SUBACHOQUE \\
\hline Peso específico & 2.56 \\
Equivalente de arena & $86 \%$ \\
Caras fracturadas & $92 \%$ \\
Índice de alargamiento & $10 \%$ \\
Índice de aplanamiento & $10 \%$ \\
Ataque en Sulfato de sodio & $12.4 \%$ \\
Microdeval (sin saturar) & $20.3 \%$ \\
Resistencia al desgaste & $22.5 \%$ \\
Máquina de los Ángeles & \\
\hline
\end{tabular}

Ensayo Marshall. Se presentan en primera instancia los resultados de las briquetas elaboradas con los dos tipos de CA convencional (CA 80-100 y CA 60-70), seguido de las muestras con CA modificado. 
Mezcla asfáltica convencional. Los cálculos obtenidos del ensayo Marshall para las briquetas elaboradas con CA convencional están registrados en las Tablas 6 y 7 . En la Tabla 6 se presentan los resultados de la mezcla convencional tipo MDC-2 que empleó CA 80-100 y en la Tabla 7, aquellas que emplearon CA 60-70.

Tabla 6. Resumen del ensayo Marshall PARA MEZCLA ASFÁLTICA CONVENCIONAL MDC-2 CON CA 80-100.

\begin{tabular}{ccccccc}
\hline \hline $\begin{array}{c}\text { \% } \\
\mathrm{CA}\end{array}$ & $\begin{array}{c}\text { Peso } \\
\text { g/cm } \mathrm{cm}^{3}\end{array}$ & $\begin{array}{c}\text { Estabilidad } \\
\text { (E) }[\mathrm{kg}]\end{array}$ & $\begin{array}{c}\text { Vacíos en } \\
\text { la mezcla } \\
{[\%]}\end{array}$ & $\begin{array}{c}\text { Vacíos en } \\
\text { agregados } \\
{[\%]}\end{array}$ & $\begin{array}{c}\text { Flujo (F) } \\
{[\mathrm{mm}]}\end{array}$ & $\begin{array}{c}\text { Relación } \\
\text { E/F } \\
{[\mathrm{kg} / \mathrm{mm}]}\end{array}$ \\
\hline \hline $4.5 \%$ & 2.21 & 710 & 7.63 & 17.52 & 3.33 & 213.09 \\
$5.0 \%$ & 2.24 & 835 & 5.76 & 16.89 & 3.45 & 242.14 \\
$5.5 \%$ & 2.24 & 853 & 5.09 & 17.33 & 3.51 & 243.48 \\
$6.0 \%$ & 2.23 & 813 & 4.64 & 17.96 & 3.70 & 219.71 \\
\hline
\end{tabular}

Tabla 7. Resumen ensayo Marshall PARA MEZCLA ASFÁLTICA CONVENCIONAL TIPO MDC-2 CON CA 60-70.

\begin{tabular}{ccccccc}
\hline $\begin{array}{c}\% \\
\mathrm{CA}\end{array}$ & $\begin{array}{c}\text { Peso } \\
\text { Unitario } \\
\mathrm{g} / \mathrm{cm}^{3}\end{array}$ & $\begin{array}{c}\text { Estabilidad } \\
\text { (E) [kg] }\end{array}$ & $\begin{array}{c}\text { Vacíos en } \\
\text { la mezcla } \\
{[\%]}\end{array}$ & $\begin{array}{c}\text { Vacíos en } \\
\text { agregados } \\
{[\%]}\end{array}$ & $\begin{array}{c}\text { Flujo (F) } \\
{[\mathrm{mm}]}\end{array}$ & $\begin{array}{c}\text { Relación } \\
\mathrm{E} / \mathrm{F} \\
{[\mathrm{kg} / \mathrm{mm}]}\end{array}$ \\
\hline $5.0 \%$ & 2.24 & 1189 & 5.83 & 16.95 & 3.40 & 349.86 \\
$5.5 \%$ & 2.25 & 1323 & 4.47 & 16.80 & 3.60 & 367.84 \\
$6.0 \%$ & 2.25 & 1393 & 3.48 & 16.96 & 3.69 & 377.18 \\
$6.5 \%$ & 2.26 & 1229 & 3.02 & 17.60 & 3.92 & 313.38 \\
\hline
\end{tabular}

Los porcentaje óptimos de CA de acuerdo con los datos de las Tablas 6 y 7 son de $5.3 \%$ y $5.6 \%$ para el caso de mezclas fabricadas con CA 80-100 y CA 60-70 respectivamente. En estos porcentajes se están cumpliendo, los requisitos mínimos exigidos por la especificación INVIAS (2007) para MDC-2 y tránsitos tipo NT1 y/o NT2 (bajos y medios volúmenes respectivamente). 
Mezclas asfálticas modificadas. Los datos de resistencia mecánica obtenidos en el ensayo Marshall para las mezclas asfálticas modificadas fueron registrados en las Tablas 8 y 9 para CA 80-100 y CA 60-70 respectivamente. Estos datos son presentados de manera gráfica en las figuras 1-3.

Tabla 8. Resumen del ensayo Marshall PARA MEZCLA ASFÁLTICA MODIFICADA CON ASFALTITA Y CA 80-100.

\begin{tabular}{|c|c|c|c|c|c|c|c|c|c|}
\hline \multirow{2}{*}{$\begin{array}{c}\% \\
\text { Asfaltita } \\
\end{array}$} & \multicolumn{3}{|c|}{ Estabilidad (E) $[\mathrm{kg}]$} & \multicolumn{3}{|c|}{ Flujo (F) $[\mathrm{mm}]$} & \multicolumn{3}{|c|}{ Relación E/F [kg/mm] } \\
\hline & $5.0 \%$ & $5.3 \%$ & $5.6 \%$ & $5.0 \%$ & $5.3 \%$ & $5.6 \%$ & $5.0 \%$ & $5.3 \%$ & $5.6 \%$ \\
\hline 0.50 & & & & 5 & & & & & \\
\hline 1.50 & 3 & & & 3.91 & & & & & \\
\hline 2.50 & & & & 4.47 & & & & & \\
\hline 3.50 & 3276 & 3834 & 2184 & 4.57 & 4.88 & 5.08 & 717.29 & 787.07 & 430.48 \\
\hline
\end{tabular}

Tabla 9. Resumen del ensayo Marshall PARA MEZCLA ASFÁLTICA MODIFICADA CON ASFALTITA Y CA 60-70.

\begin{tabular}{c|c|c|c|c|c|c|c|c|c}
\hline \multirow{2}{*}{$\begin{array}{c}\text { \% } \\
\text { Asfaltita }\end{array}$} & \multicolumn{2}{|c|}{ Estabilidad (E) [kg] } & \multicolumn{3}{c|}{ Flujo (F) $[\mathrm{mm}]$} & \multicolumn{3}{c}{ Relación E/F [kg/mm] } \\
\cline { 2 - 11 } & $5.3 \%$ & $5.6 \%$ & $5.9 \%$ & $5.3 \%$ & $5.6 \%$ & $5.9 \%$ & $5.3 \%$ & $5.6 \%$ & $5.9 \%$ \\
\hline 0.50 & 1866 & 1784 & 1998 & 3.62 & 2.99 & 4.32 & 517.37 & 597.89 & 463.30 \\
1.50 & 2591 & 2545 & 2349 & 4.45 & 3.65 & 3.87 & 583,70 & 698.02 & 606.97 \\
2.50 & 2884 & 2931 & 3037 & 4.83 & 4.16 & 5.02 & 598.45 & 705.82 & 606.45 \\
3.50 & 3219 & 2931 & 2392 & 6.03 & 4.83 & 5.46 & 534.09 & 607.55 & 439.14 \\
\hline
\end{tabular}

En la figura 1a y c se observa que los valores de estabilidad $(E)$ y relación entre la estabilidad y el flujo $(F)$ (llamada por algunos investigadores como rigidez Marshall y físicamente puede ser entendida como una resistencia mecánica evaluada en el estado de falla de las mezclas, bajo carga monotónica en un ensayo de tracción indirecta) de las mezclas modificadas que emplean CA 80-100 son mayores, para cualquier porcentaje de CA y asfaltita, en comparación con la mezcla convencional. Los valores de estabilidad aumentan cuando se incrementa el contenido de asfaltita entre $0.5 \%-2.5 \%$ para contenidos de CA de $5.0 \%, 5.3 \%$ y $5.6 \%$. 
Para el caso de $3.5 \%$ de adición de asfaltita la estabilidad propende a conservar su máximo valor y la tendencia es a disminuir. Los mayores valores de estabilidad y resistencia mecánica bajo carga monotónica (evaluada a través de la relación $E / F$ ) se obtienen cuando se adiciona la asfaltita al contenido óptimo de CA de $5.3 \%$ determinado del ensayo Marshall, seguido por las mezclas fabricadas con $5.0 \%$ y $5.6 \%$ de CA. La mayor resistencia mecánica de las mezclas $(946.61 \mathrm{~kg} / \mathrm{mm}$ ) se obtiene cuando se adiciona $0.5 \%$ de asfaltita al 5.3\% de CA. Esta resistencia es 3.84 veces mayor que aquella que alcanza la mezcla convencional. La evolución y los valores de flujo de las mezclas son similares para cualquier porcentaje de CA y asfaltita (ver figura 1b). Las mezclas modificadas experimentan mayores flujos que las mezclas convencionales debido principalmente a que para llevarlas a la falla es necesario deformarlas más (debido a su mayor resistencia mecánica bajo carga monotónica a tracción indirecta o a la estabilidad).

Las mezclas modificadas que emplean CA 60-70 presentan un comportamiento similar a aquellas fabricadas con CA 80-100. En la figura 2 a y c se observa que los valores de estabilidad y resistencia de las mezclas modificadas que emplean CA 60-70 son mayores, para cualquier porcentaje de CA y asfaltita, en comparación con la mezcla convencional. Los valores de estabilidad aumentan cuando se incrementa el contenido de asfaltita entre $0.5-2.5 \%$ para contenidos de CA de 5.3, 5.6 y 5.9\%. Para el caso de 3.5\% de adición de asfaltita la estabilidad tiende a conservar su máximo valor y la tendencia es a disminuir. Los mayores valores $E / F$ se obtienen cuando se adiciona la asfaltita al contenido óptimo de CA de $5.6 \%$ determinado del ensayo Marshall. La mayor resistencia bajo carga monotónica de las mezclas $(705.82 \mathrm{~kg} / \mathrm{mm})$ se obtiene cuando se adiciona $2.5 \%$ de asfaltita al $5.6 \%$ de CA. Esta resistencia es 1.89 veces mayor que aquella que alcanza la mezcla convencional.

Para cualquier porcentaje de CA, los valores de resistencia incrementan cuando se adiciona la asfaltita en porcentajes de $0.5 \%$ a $1.5 \%$, luego dicha resistencia se estabiliza en $2.5 \%$ de asfaltita para luego disminuir su valor cuando se adiciona $3.5 \%$ (ver figura 
2c). La evolución y los valores de flujo de las mezclas son similares para cualquier porcentaje de CA y asfaltita (ver figura $2 b$ ). De igual forma que las mezclas modificadas con CA 80-100, Las mezclas modificadas con CA 60-70 experimentan mayores flujos que las mezclas convencionales.

a)

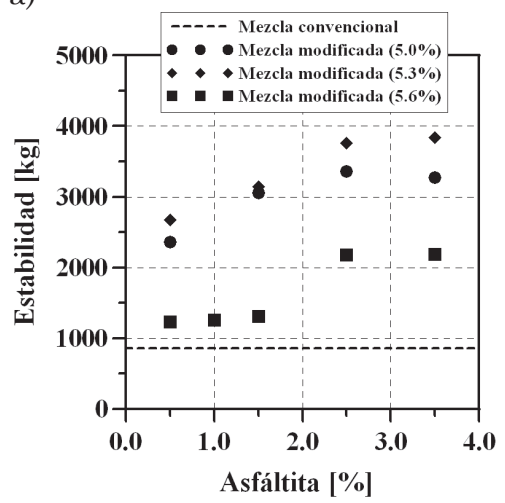

b)

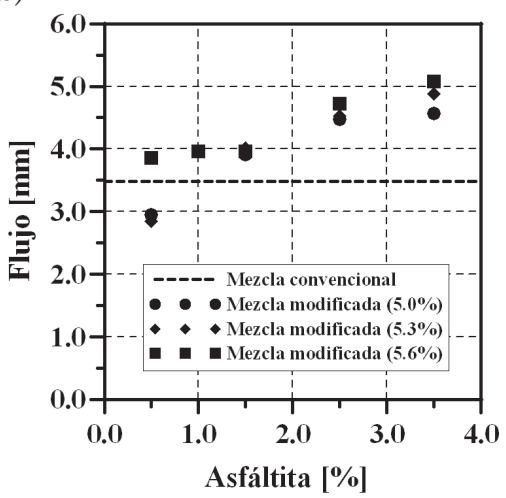

c)



Figura 1. a) Estabilidad, b) flujo y C) Rigidez Marshall vs. Porcentaje de ASFALTITA PARA MEZCLAS MODIFICADAS MDC-2 EMPLEANDO CA 80-100.

Las mezclas asfálticas modificadas con asfaltita, experimentan un incremento notable en la resistencia mecánica en comparación con las mezclas convencionales (ver figura 3c). Comportamientos similares han sido obtenidos modificando el cemento asfáltico con 
otros aditivos poliméricos del tipo plastómero (Rondón et al, 2004; Rondón et al, 2006; Rondón et al, 2007; Rondón et al, 2009). A pesar de que el CA 80-100 es de mayor penetración y menor rigidez que el CA 60-70, es interesante observar en la figura 3 a y c que los mayores valores de estabilidad y $E / F$ se obtienen cuando se modifican con asfaltita las mezclas que emplean CA 80-100. Lo anterior será objeto de investigaciones en un futuro, pero un comportamiento similar fue reportado por Toro \& Amortegui, (2008) cuando se modificaron mezclas asfálticas con desecho de policloruro de vinilo (PVC) empleando el mismo agregado pétreo y los mismos CA.

a)

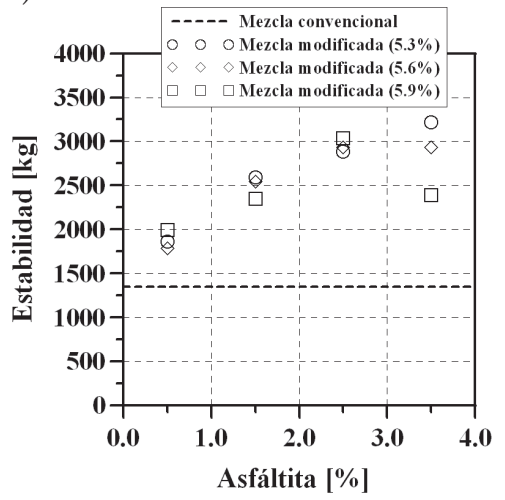

b)

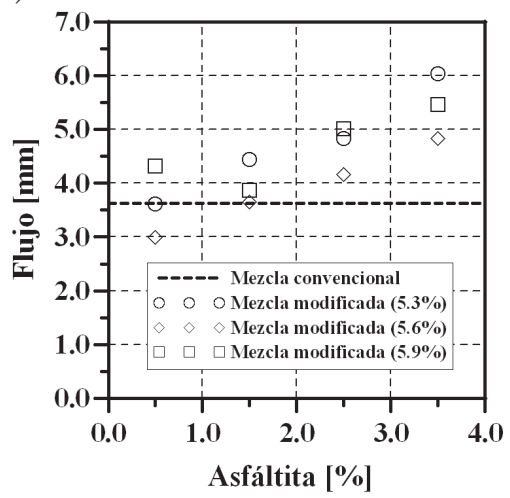

c)

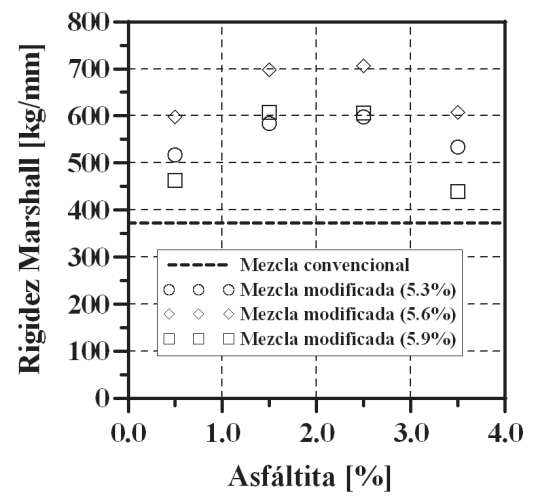

Figura 2. a) Estabilidad, B) flujo y C) Rigidez Marshall vs. Porcentaje de ASFALTITA PARA MEZCLAS MODIFICADAS MDC-2 EMPLEANDO CA 60-70. 


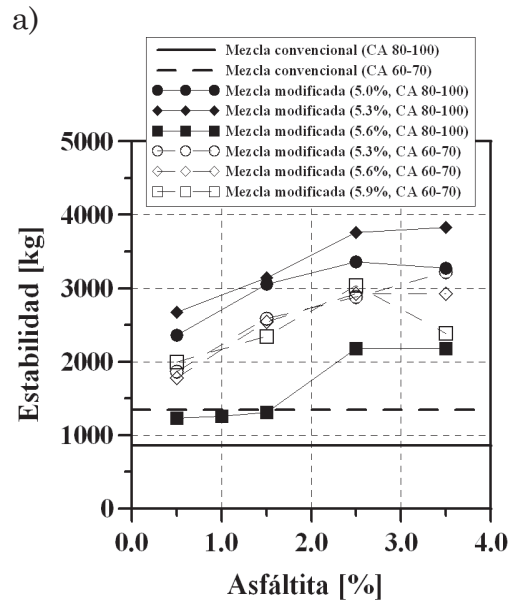

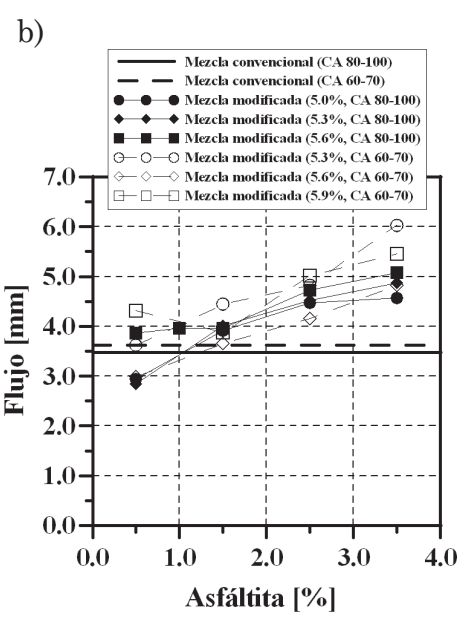

c)

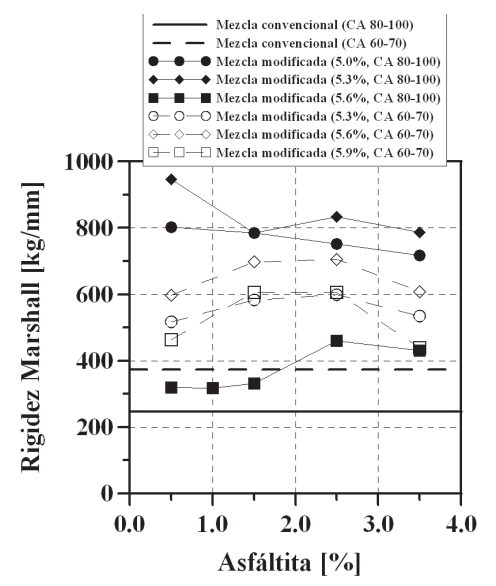

Figura 3. A) Estabilidad, B) flujo y c) Rigidez Marshall vs. Porcentaje de asfaltiTA PARA MEZCLAS MODIFICADAS MDC-2 EMPLEANDO CA 80-100 y CA 60-70.

Módulo resiliente y deformación permanente. En las figuras 4 y 5 se presenta la evolución del módulo resiliente y la deformación permanente en la dirección vertical de las mezclas asfálticas modificadas con asfaltita. Se observa un incremento típico del módulo cuando se aumenta la frecuencia de carga y disminuye la temperatura del ensayo. La rigidez de las mezclas 
incrementa notablemente $\mathrm{y}$, por lo tanto, la resistencia a la deformación permanente, cuando se adiciona asfaltita al CA.

Para el caso de temperatura de ensayo de $10^{\circ} \mathrm{C}$ el módulo de las mezclas modificadas incrementa entre un 51\% - 113\% con respecto a la mezcla convencional cuando se adiciona asfaltita entre $0.5 \%$ - $3.5 \%$ respectivamente. Para temperatura de $20^{\circ} \mathrm{C}$ y $30^{\circ} \mathrm{C}$ estos incrementos son de $63 \%$ - 286\% y $108 \%$ - $678 \%$ respectivamente. La deformación permanente disminuyó entre 9.03\% - 63.5\% cuando se adicionó asfaltita entre $0.5 \%-3.5 \%$ respectivamente.

Adicionalmente, se observa que la mezcla convencional no cumple el valor máximo de deformación que se exige para el ensayo realizado, el cual es de $1.0 \%$ medido a $3 \times 10^{3}$ ciclos de carga. Sin embargo, cuando se modifican las mezclas con asfaltita la tendencia es a mejorar esta propiedad y cuando se adiciona $3.5 \%$ el valor es muy cercano al permitido. Este aumento en rigidez y resistencia a la deformación permanente se debe a que con la incorporación de la asfaltita al $\mathrm{CA}$ se obtiene un material más rígido y viscoso tal como se observa en el subcapítulo siguiente.

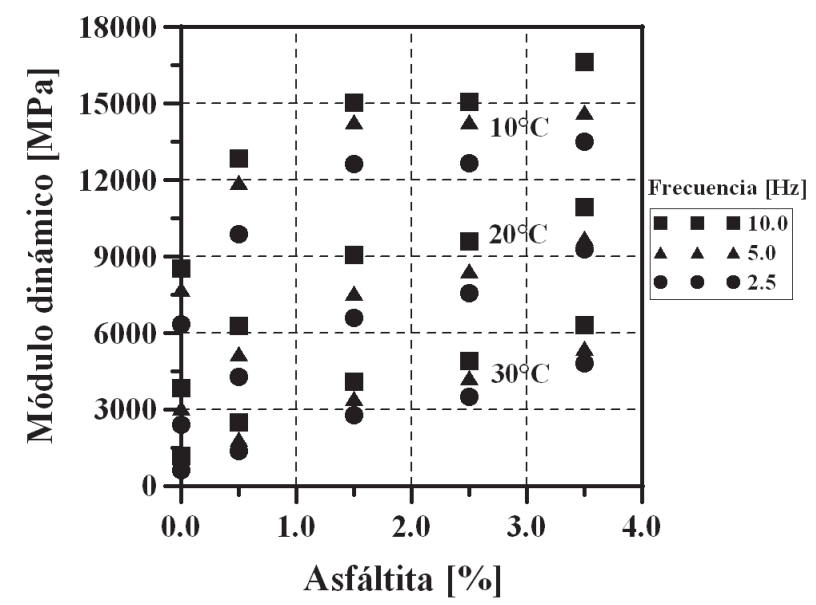

Figura 4. Evolución del módulo Resiliente vs. Porcentaje de asfaltita para MEZCLAS MODIFICADAS MDC-2 EMPLEANDO CA 80-100. 


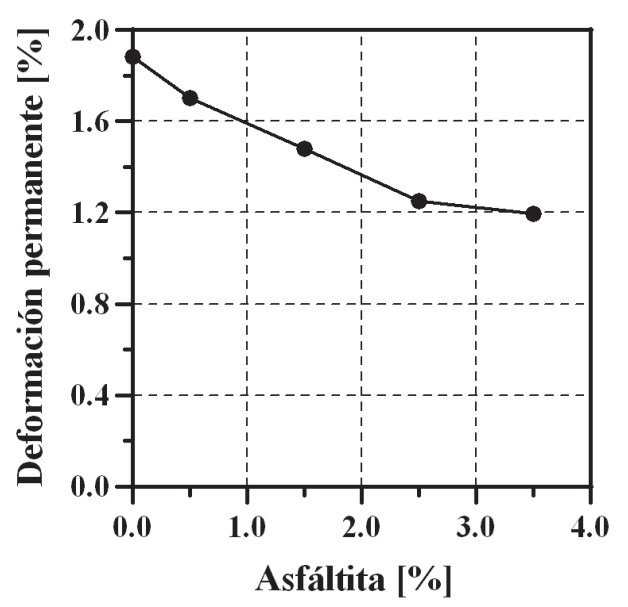

Figura 5. Evolución de La deformación PeRmanente Vertical Vs. Porcentaje de ASFALTITA PARA MEZCLAS MODIFICADAS MDC-2 EMPLEANDO CA 80-100.

Caracterización del asfalto. En la figura 6 a y b se presenta la evolución de la penetración con la temperatura para los asfaltos convencionales (CA 80-100 y CA 60-70) y modificados. Se observa de manera general que los asfaltos modificados son menos penetrables para cualquier porcentaje de CA y asfaltita en comparación con los convencionales. Cuando se adiciona asfaltita en porcentajes entre $2.5 \%$ y $3.5 \%$ al CA, el cemento asfáltico modificado se rigidiza a tal punto que el grado de susceptibilidad térmica es muy pequeño y, en este rango de adición, la penetración en ambos tipos de asfalto es similar (ver figura 6).

En la figura 7 se presenta la evolución del punto de ablandamiento con el porcentaje de asfaltita adicionado a los CA. Se observa un incremento aproximadamente lineal de esta propiedad con la adición de asfaltita lo que significa que los CA modificados presentan una mayor resistencia a fluir (viscosidad) y consistencia en comparación con el CA sin modificar. Adicionalmente, se observa que el CA 80-100 tiende a incrementar mucho más rápido el punto de ablandamiento (y por lo tanto su viscosidad) cuando se modifica con asfaltita, en comparación con el CA 60-70. Para entender los cambios que ocurren en las propiedades físicas de 
los CA modificados es necesario evaluar la composición química de los mismos.

a)

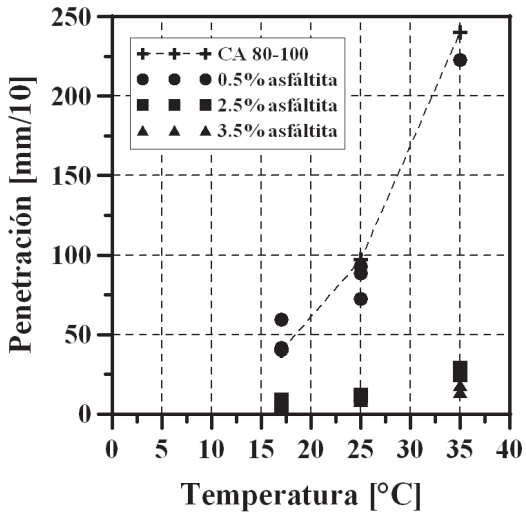

b)

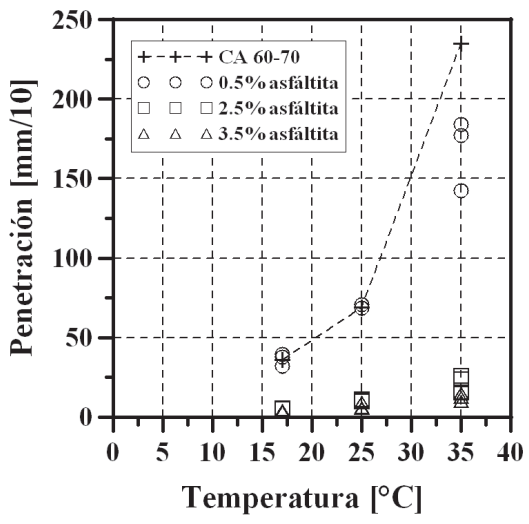

Figura 6. Penetración Vs. temperatura del ensayo PARA A) CA 80-100 Y B) CA 60-70.

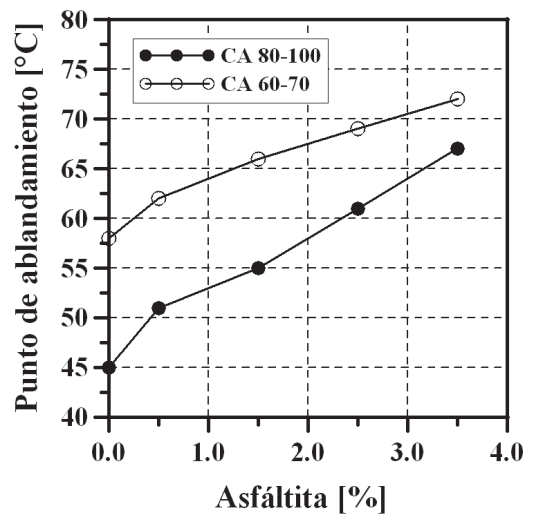

Figura 7. Evolución punto de ablandamiento vs. Porcentaje de asfaltita PARA MEZCLAS MODIFICADAS MDC-2 EMPLEANDO CA 80-100. 


\section{Conclusiones}

En general, las mezclas asfálticas modificadas con asfaltita tienden a poseer un comportamiento rígido. A bajas temperaturas de servicio estas mezclas pueden tener un comportamiento frágil, llevando a pensar que tendrían un mejor desempeño en climas cálidos.

Los valores de estabilidad y rigidez Marshall de las mezclas modificadas con CA 80-100 y CA 60-70 son mayores, para cualquier porcentaje de CA y asfaltita, en comparación con la mezcla convencional. Los mayores valores de estabilidad y rigidez se obtienen cuando se modifican con asfaltita las mezclas que emplean CA 80-100, a pesar que este tipo de asfalto presenta menor resistencia a la penetración y rigidez que el CA 60-70.

Los módulos dinámicos de las mezclas modificadas con CA 80-100 son superiores a aquellos alcanzados por las convencionales, y los mayores incrementos se obtienen cuando la temperatura del ensayo aumenta. Lo anterior permite prever que la asfaltita como modificador de asfaltos puede ser un material que permita mejorar las características de rigidez y resistencia a las deformaciones permanentes de mezclas que sean utilizadas en climas cálidos.

La resistencia que tienen los asfaltos modificados a fluir es mayor con respecto al convencional. Los valores de penetración del asfalto modificado permiten predecir menor ahuellamiento a altas temperaturas de servicio en comparación con los convencionales. Cuando se adicionan porcentajes de asfaltita entre 2.5 y $3.5 \%$ a los CA 80-100 y CA 60-70, ambos tipos de asfaltos experimentan valores similares en el ensayo de penetración.

Las fases futuras del proyecto deben medir propiedades como envejecimiento a corto y largo plazo de los asfaltos y resistencia a fatiga de las mezclas asfálticas. Adicionalmente, para entender los cambios que ocurren en las propiedades físicas y mecánicas de las mezclas modificadas es necesario evaluar los cambios que experimentan las propiedades químicas de las mismas. 


\section{REFERENCIAS}

Copeland, A. R., Youtcheff Jr, J. S \& Shenoy, A. (2007). Moisture Sensitivity of Modified Asphalt Binders: Factors Influencing Bond Strength. Transportation Research Record: Journal of the Transportation Research Board, Issue number 1998, pp. 18-28.

MINTRANSPORTE - Ministerio de Transporte - Oficina de Planeación (2000). Parque Automotor de Transporte de Carga en Colombia. Bogotá D.C., Colombia.

MINTRANSPORTE - Ministerio de Transporte - Oficina de Planeación (2004). El Transporte en Cifras. Bogotá D.C., Colombia.

INVIAS - Instituto Nacional de Vías (2007). Especificaciones generales de Construcción de carreteras. Bogotá D.C (Colombia).

INVIAS - Instituto Nacional de Vías. (1997). La asfaltita como modificador de los asfaltos colombianos. Modificación de asfalto de la refinería de Cartagena con asfaltita colombiana. Laboratorio Central del Instituto Nacional de Vías, 33 pp.

INVIAS - Instituto Nacional de Vías (2007a). Normas de Ensayos de Materiales para Carreteras. V. I y II. Bogotá D.C (Colombia).

Papagiannakis, A. T. \& Lougheed, T. J. (1995). A review of crumb-rubber modified asphalt concrete technology. Research report for project T990209 "Rubber-Asphalt Study", Washington State Transportation Commission and U. S. Department of Transportation.

Rondón, H. A., Herrera, O. A., Caicedo, L. R., Díaz, D. M., Gutiérrez, A. P., Ladino, C. M. \& Díaz, F. A. (2004). Estudio del comportamiento de mezclas densas en caliente elaboradas con asfaltos modificados con policloruro de vinilo, látex natural y caucho reciclado. En 4as. Jornadas Internacionales del Asfalto, Cartagena, $17 \mathrm{pp}$.

Rondón, H. A., Rodríguez, E., Mojica, A. A. \& Hernández, H. (2006). Análisis del comportamiento de una mezcla densa en caliente (MDC-1) modificando el asfalto con desecho de polietileno. En 5as. Jornadas Internacionales del asfalto, Cartagena, $12 \mathrm{pp}$.

Rondón, H. A, Rodríguez, E. \& Moreno, L. A. (2007). Comportamiento de mezclas densas en caliente elaboradas con asfaltos modificados con desechos de policloruro de vinilo (PVC), polietileno de alta densidad (PEAD) y poliestireno (PS). Revista Ingenierías Universidad de Medellin, No. 11, 91-104. 
Rondón, H. A., Reyes, F. A., Figueroa, A. S., Rodríguez, E., Real, C. M. \& Montealegre, T. A. (2008). Mezclas asfálticas modificadas en Colombia. Revista Infraestructura Vial, No. 19, 12-21.

Rondón, H. A., Reyes, F. A. \& Ojeda, B. E. (2009). Comportamiento de una mezcla densa en caliente modificada con desecho de policloruro de vinilo (PVC). Revista Ciencia e Ingeniería Neogranadina (aceptado para publicación).

Toro, E. \& Amortegui, M. A. (2008). Influencia del tipo de asfalto en el comportamiento de un concreto asfáltico modificado con desecho de PVC. Tesis de Pregrado, Facultad de Ingeniería Civil, Universidad Católica de Colombia, 79 pp. 